\title{
Brain Tumor Detection through MR Images: A Review of Segmentation Techniques
}

\author{
Madhvi Arya \\ M.Tech Scholar \\ ECE, UCOE \\ Punjabi University, India
}

\author{
Reecha Sharma \\ Assistant Professor \\ ECE, UCOE \\ Punjabi University, India
}

\begin{abstract}
In this paper, a review on computer-based methods which defines the tumor region in the brain using MRI images is discussed. Various image processing techniques are reviewed in this paper which helps to enhance the images for the computerized detection of brain tumor. Magnetic Resonance Imaging (MRI) is mostly used to obtain medical imaging with very high quality. MRI is a very advanced technique which provides very rich information about size, shape and location of brain tumors without any need to expose the patient to a high ionization radiation. In medical imaging, segmentation is used to segment abnormal tissues from normal tissues and reliable, accurate, and automatic segmentation of these structures and tissues can results in improved diagnosis and treatment of disease. Digital Image Processing provides number of methods to study bio-medical images from different aspects. The paper focuses on to provide overview for different segmentation techniques involved in brain tumor detection.
\end{abstract}

\section{Keywords}

Magnetic resonance imaging (MRI), Image segmentation, Digital Image Processing (DIP)

\section{INTRODUCTION}

Digital Image processing is a growing field which helps in Classification, Feature extraction, Multi-scale signal analysis, Pattern recognition, Projection, enhancement of an image, in order to fetch useful information from that image, which provides an easy pathway to doctors for the evaluation of complex disease such as kidney stones, cancer, breast cancer, brain tumor etc[11]. It becomes very difficult task to segment the brain images manually and the probability of error percentage is also very high which results in large number of disabilities and death worldwide. The technique which is mainly used for tumor detection is MRI [4]. MRI technology makes use of train pulses of radio wave energy and magnetic field that makes images of organs and structures within a body. In order to process these MRI's, various DIP techniques included are Image editing, Images Enhancement and Filtering, Linear filtering, Pixelation.

The paper is organized as follows: firstly, there is brief introduction about various pre-processing techniques. In next section, we discuss about the segmentation techniques involved and after this there will be evaluation, analysation and validation of different segmentation algorithm. Finally conclusions are addressed for different segmentation techniques. The algorithm followed to detect brain tumor is as follows:

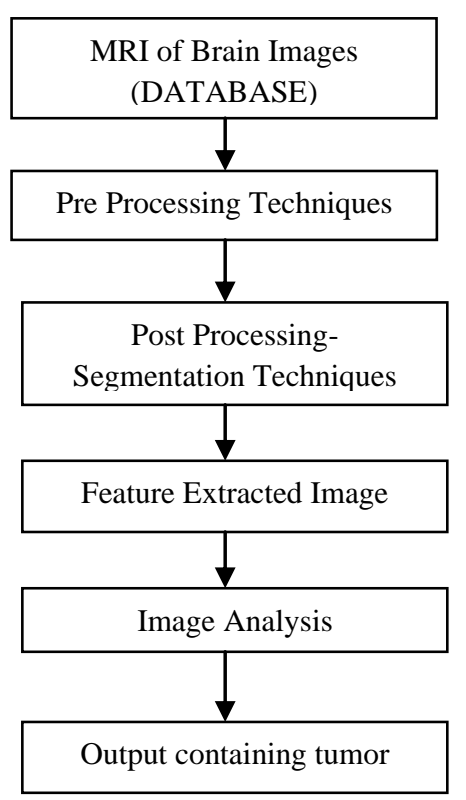

Fig 1: Steps for brain tumor detection

\section{LITERATURE REVIEW}

Kusum Rani et al. [8] presented a review on various image fusion techniques like simple average, simple maximum, simple minimum, DWT, PCA and then concluded that combination of PCA and DWT can be moulded further for better results. W. M. Wells et al. (1996) [4] presents an automatic method adaptive segmentation for correcting the intensity of MRI images. This technique increases the automation level and improves the 3-D reconstruction for disease research, planning of surgery, evaluation of therapy and other purposes. Ilya Pollak Willsky et al. (2000) [19] introduced differential equations of first-order having multiple dimensions. It demonstrates the applicability of these equations for the processing of images. Y. Zhang et al.(2000) [9] presented a method for the classification of MR image as normal or abnormal. In this paper firstly features are extracted from image using wavelet transformation, then in order to reduce dimension of extracted features, principle component analysis is used and are passed to support vector machine (KSVM). They uses four different kernels HPOL, LIN, IPOL and GRB to increase the accuracy and robustness of their classifier. Bing Nan Li et al. (2011) [10] presented a new fuzzy level set algorithm. This method is further modified with regular local evaluation. They facilitated the manipulation level set to extract the boundaries of region of interest resulting in reduction of manual intervention. 


\section{PRE-PROCESSING TECHNIQUES}

The MRI images obtained may not be of very good quality which is required for analysation. Images may be noisy, blurred, Low contrast. Region of interest might become difficult to extract [14]. To increase the accuracy of different segmentation techniques, the image needs to be preprocessed, so that tumor location is correctly identified. Several pre-processing operations involved are filtration, denoising methods, intensity normalization.

\subsection{Image Filtration Methods}

Filters described below are Low pass Filter, Median Filter, Mean Filter, Gaussian Filter, High pass filter.

In low pass filter, only low intensity value of the pixels are passed, then discrete Fourier transform of the image is computed. An image operator is applied to the transformed image and the result which is obtained after applying image operator is computed with inverse Fourier transform. Threshold value is given by Fourier transform. All the values which are above threshold frequency are suppressed by LPF and smaller values remain unchanged. This can be written in equation as [16]

$$
\begin{gathered}
\mathrm{H}(\mathrm{u}, \mathrm{v})=D(u, v) \quad D(u, v) \leq D_{0} \\
0 \quad D(u, v)>D_{0} \\
D(u, v)=\left[(u-M / 2)^{2}+(v-N / 2)^{2}\right]^{1 / 2}
\end{gathered}
$$

where $\mathrm{H}=$ different values which pixels can adopt.

Gaussian Filter, to prevent wrong detection caused by noise it is necessary to remove the noise. Gaussian filter convolved with the image to smoothen it.

Median Filter removes the noise while it preserves the edges and it also reduces salt and pepper noise. The new value of pixel after removal of noise is calculated by sorting all values of the surrounding pixels by their size. Middle value from the sorted series is assigned as new value.

$\operatorname{Median}[\mathrm{A}(\mathrm{x})+\mathrm{B}(\mathrm{x})] \neq \operatorname{median}[\mathrm{A}(\mathrm{x})]+\operatorname{median}[\mathrm{B}(\mathrm{x})]$

where $\quad \mathrm{A}(\mathrm{x})$ and $\mathrm{B}(\mathrm{x})=$ pixel values of images

In High pass Filter only high intensity value of the pixels are passed. All the values which are less than threshold frequency are suppressed by HPF and larger values remain unchanged.

Mean Filter is a sliding window filter, in which central value of window is replaced by average of all values present in the window. The basic function for mean is [16]

$$
f(x, y)=\frac{1}{k} \sum_{i=1}^{k} g(x, y)
$$

where $\quad f(x, y)=$ output mean

$$
\mathrm{g}(\mathrm{x}, \mathrm{y})=\text { original values }
$$

the size of window depends upon value of $\mathrm{K}$. if value of $\mathrm{K}$ is one then size of window is $3 \times 3$ and position of centre is $2 \times 2$, which is replaced by average value of pixels present in the window. For example, if intensity values are 40, 55, 51, 46, $43,55,57,45,58$ before filtering. So the average value is 50 and then 43 are replaced by 50 .

\begin{tabular}{|c|c|c|}
\hline 40 & 55 & 51 \\
\hline 46 & 43 & 55 \\
\hline 57 & 45 & 58 \\
\hline
\end{tabular}

\begin{tabular}{|c|c|c|}
\hline 40 & 55 & 51 \\
\hline 46 & 50 & 55 \\
\hline 57 & 45 & 58 \\
\hline
\end{tabular}

Fig 2: Window of size $3 * 3$ and second window showing pixel replaced by average value.

\subsection{De-noising methods}

Methods used for removing noise are anisotropic filter, nonlinear filter, wavelet models, a Markov random field (MRF) models, non-local means models.

Nonlinear Filter is used for the removal of non-additive noise. They examine the window which is surrounding each sample, and implement some suitable algorithm in order to get suitable value for that pixel.

Wavelet Models are mathematical functions, helps to recover weak signals from noise in signal processing .This technique is mainly used in medical applications. After analysing an image, wavelet compression algorithm works and is converted into number of mathematical expressions that can further be decoded by the receiver.

Markov random field (MRF) models A noisy image with some missing measurements are applied to this model with the aim to recover original image. The isolated voxels are removed of one tissue class which are considered not to be the member of this tissue type. Holes of one tissue type also get closed in a cluster of connected voxels. As a result the noise level will reduce in segmentation.

Non-Local means models this algorithm takes the mean of pixels which are surrounding the pixel needs to be replaced. In particular, the weightage for a pixel is based on the degree of similarity between a small patch centred on that pixel and the small patch centred on the pixel being de-noised.

\section{SEGMENTATION TECHNIQUES}

It is the process of partitioning an image into super-pixels. The aim of segmentation is to represent an image into more meaningful and simplified form which results in easy analyzation. It helps to locate lines, objects, curves and boundaries in images. In the process of segmentation, a label is assigned to each pixel, and pixels with same labels define common characteristics. The segmented parts obtain covers the whole image. Main applications of segmentation in medical imaging is to study anatomical structure, Surgery planning, Locate tumors, Intra-surgery navigation, Measure tissue volumes, Virtual surgery simulation [5].

\subsection{Clustering Based Segmentation}

It comprises of K-means and fuzzy k-mean clustering. These techniques are investigated using squared difference and absolute difference.

$K$-mean clustering the algorithm flows as, k data points are selected which act as centres of clusters. These points are continuously selected till the centres remain unchanged. It is given by

$$
\arg \min \sum_{i=1}^{k} 1 \sum_{x \in S_{i}} 1\left(\left|x-\mu_{i}\right| 2^{2}\right)
$$

where $\mathrm{S}=$ observational sets.

$$
\mu_{i}=\text { mean of points in } S_{i}
$$


Data point is allocated to the cluster which is having nearest centre. Now every cluster is checked for at least one data point. If some cluster appears to be empty then a randomly chosen point is assigned to that cluster and cluster centres are replaced by mean of cluster elements. As a result of this objects get separated into groups and metric can be calculated.

Fuzzy C-mean clustering, in this clustering data can belong to two or more than two clusters and the way, how data is bounded to each cluster is defined by membership functions [1]. A high degree of membership is assigned to those datapoint which lies near to the centre of a cluster and low degree of membership is assigned to those which lie away from centre of cluster. The use of fuzzy c-mean is in the field of bioinformatics to study gene expressions. Genes with same expressions are clustered into same group and different clusters will have genes with distinct characteristics.

\subsection{Histogram Based Segmentation}

In this segmentation technique, all pixels of image are used to create histogram. The gray levels of the histogram represent background and the object of image. The first largest peak defines the background and the second largest peak defines the object. After this threshold point is selected between the two peaks in the valley and image is thresholded. Thresholding sets that pixel to one whose value is on the object side and all other values are set to zero [3]. The keys for histograms are peaks and the valley between them. But sometimes it may become difficult to identify significant valleys and peaks. To overcome this problem two steps involved are histogram equalization and histogram smoothing. Equalization results into an image of better contrast and smoothening results in removing spikes while maintaining the basic shape of histogram.

\subsection{Region Growing Method}

It comprises of seeded region growing method, unseeded region growing method, $\lambda$-connected segmentation. This technique is based on the assumption that one region consists of similar pixel values.

\section{Seeded Region Growing Method}

In this method set of seeds are taken along with the image and each of the object is marked segmented by the seeds. Then unallocated neighbouring pixels are continuously compared with region, which results in growth of region [12]

In order to check whether pixel belongs to particular region or not the difference between pixel intensity value and region mean is used. The pixels are assigned to their respective regions according to the difference measured. This process is repeated again and again till all pixels are allocated to a region. Because seeds are taken as input, all outputs depend upon the selection of seeds, and if the noise is present in the image it can cause poor placements of seeds.

\section{Unseeded Region Growing Method}

This method is the modified form of seeded growing method. In this method explicit seeds are not required. In this if the difference measured is less than threshold value then this pixel is assigned to respective region, else pixel is assigned to a new region having consideration that it differs from all present regions.

\section{$\lambda$-connected segmentation}

This segmentation is based on linking paths between the pixels and their intensities. The path which is formed by pixels defines the degree of connectivity. The degree of connectivity is said to be $\lambda$ if there exists path between two pixels and connectedness is at least $\lambda$.

\subsection{SVM based Segmentation}

This segmentation method segments the healthy tissues, subcompartments of healthy and tumor regions and has the ability to automatically learn the nonlinear distribution of the image data by making the use of implicit learning kernel and by SVM parameters training. After this another method is proposed which segments only one tumor region. To improve this, multi kernel method is approached which segments the brain tumor from multi-sequence MRI images. When multi kernel is compared with single kernel, this method improves the accuracy, reduces error, increases speed by regularizing different stages and hence improves result.

\subsection{MRF based Segmentation}

Clustering methods reduces the effect of noise and problems of overlapping on the result. MRF checks out a region as tumor or non-tumor area, and simultaneously verifies if the neighbouring region also classify the same or different [2]. This algorithm mainly depends upon data sets to calculate results of brain tumor segmentation. If lower layers are having similar characteristics, then voxel will evolve itself to higher classification. Further a correspondence is ensured between patient image and atlas, before going for registration step. the tumor growth model is prepared to rectify MRF energy minimization problem. This method is simple, nonparametric, and fast compared to other approaches while maintaining similar accuracy.

\subsection{Watershed Segmentation}

This segmentation is operating tool based on mathematical morphology and is a very good technique to separate tumor region from the image.

In watershed segmentation magnitude of an image which is gradient is considered as a topographic surface. Region boundaries are defined by those pixels which are having highest gradient magnitude intensities and these pixels corresponds to watershed lines. It is assumed as water is placed on pixel as is surrounded by watershed lines flowing from top to the bottom of hill considered as local intensity minimum. A catch basin is formed by pixels which are draining to minimum form.

For an image $\mathrm{f}(\mathrm{x}, \mathrm{y})$, following two equations are used to calculate the angle and gradient vector magnitude at which intensity level varies to maximum range. Sobel mask is used to calculate magnitude of the gradient.

$$
\begin{aligned}
& \mathrm{g}(\mathrm{x}, \mathrm{y})=\sqrt{ }\left(\mathrm{g}_{1}{ }^{2}(\mathrm{x}, \mathrm{y})+\mathrm{g}_{2}{ }^{2}(\mathrm{x}, \mathrm{y})\right) \\
& \alpha(\mathrm{x}, \mathrm{y})=\tan ^{-1}\left(\frac{g_{2}(x, y)}{g_{1}(x, y)}\right)
\end{aligned}
$$

Where $\mathrm{g}_{1}(\mathrm{x}, \mathrm{y}), \mathrm{g}_{2}(\mathrm{x}, \mathrm{y})=$ gradients in the $\mathrm{x}, \mathrm{y}$ direction.

\section{CONCLUSION}

The main drawback of k-mean clustering sometimes it becomes difficult to predict the value of $\mathrm{K}$ and this technique does not produce good results for clusters of different density and different sizes.

Fuzzy c-means clustering does not work well with high dimensional data sets, with number of prototypes and results are also not accurate if the distance of data objects for all distributions and from all points of view seems to be equally distant. 
In histogram based segmentation, sometimes it becomes difficult to get information about how much data is quantified in an interval and also results in under segmentation and over segmentation problem. Region growing based segmentation does not provide global view of the problem. It is an expensive and noise sensitive technique.SVM based segmentation provides better results for brain tumor segmentation as compared to fuzzy clustering [6].
Markov Random Field Model can accurately extract object configurations from real and synthetic images. But this model does not provide separate entity for the objects which are touching or overlapping with each other. The watershed transformation tool results in over segmentation problem, though this problem can be overcome with aid of other segmentation technique.

Table 1. Comparison of Classification Accuracy Obtained by Various Methods

\begin{tabular}{|c|c|c|c|c|}
\hline Author & De-noising Method & Segmentation Technique Used & \multicolumn{2}{|c|}{ Accuracy in \% } \\
\hline \multirow[t]{2}{*}{ M. Madheswaran et al.[21] } & \multirow[t]{2}{*}{ ORNRAD filter } & \multirow[t]{2}{*}{ Support Vector Machine } & SVM-Linear & 95.34 \\
\hline & & & SVM Polynomial & 96.51 \\
\hline \multirow[t]{2}{*}{$\begin{array}{c}\text { Eltaher Mohamed Hussein } \\
\text { et al. [7] }\end{array}$} & \multirow[t]{2}{*}{$\begin{array}{c}\text { Adaptive filter \& } \\
\text { Enhancement filter }\end{array}$} & \multirow[t]{2}{*}{ Artificial Neural Networks } & $\begin{array}{l}\text { Recurrent Network } \\
\text { (RNN) Design }\end{array}$ & 76.47 \\
\hline & & & Elman Network Design & 88.24 \\
\hline \multirow{3}{*}{$\begin{array}{l}\text { Eman Abdel-Maksoud et al. } \\
{[18]}\end{array}$} & \multirow[t]{3}{*}{ Median filter } & \multirow[t]{3}{*}{ Hybrid clustering technique } & K-means & 80 \\
\hline & & & Mean Shift & 85.7 \\
\hline & & & KIFCM & 90.5 \\
\hline \multirow[t]{4}{*}{ B. Sathees et al. [15] } & \multirow[t]{4}{*}{ Gaussian Filter } & \multirow[t]{4}{*}{ Image Mining Techniques } & Intensity histogram & 89 \\
\hline & & & GLCM & 95.25 \\
\hline & & & $\mathrm{J} 48$ & 97.50 \\
\hline & & & Intensity & 93 \\
\hline \multirow[t]{2}{*}{ Harneet Kaur et al. [13] } & \multirow[t]{2}{*}{ Median filter } & \multirow[t]{2}{*}{ Object Based Segmentation } & Neural & 78 \\
\hline & & & Fuzzy neurone & 94 \\
\hline \multirow[t]{4}{*}{ Arashdeep Kaur [11] } & \multirow[t]{4}{*}{ High pass Filter } & \multirow{4}{*}{$\begin{array}{c}\text { Otsu's method } \\
\text { Thresholding method } \\
\text { K-means method } \\
\text { Fuzzy c-means method }\end{array}$} & K-means & 89.01 \\
\hline & & & Otsu's Method & 89.88 \\
\hline & & & Fuzzy C-means & 90.57 \\
\hline & & & Thresholding & 87.32 \\
\hline
\end{tabular}

\section{REFERENCES}

[1] Roland Winkler, Frank Klawonn, Rudolf Kruse," Problems of Fuzzy c-Means Clustering and Similar Algorithms with High Dimensional Data Sets”, 2012.

[2] Ekta Sharma, Nidhi Seth, "A Survey on the MRF Model Using Image Segmentation Techniques", In IJARIIE, Vol-2 Issue-3, 2016.

[3] Julie DELON, Agnes DESOLNEUX, Jose-Luis LISANI, Ana-Belen PETRO, "A non-parametric approach for histogram segmentation", 2011

[4] W. M. Wells, W.E.L Grimson, R. Kikinis, F.A. Jolesz, "Adaptive Segmentation of MRI Data", In IEEE transactions on Medical Imaging, volume 15, No. 4, august 1996.
[5] Jin Liu, Min Li, Jianxin Wang, Fangxiang Wu, Tianming Liu, and Yi Pan, "A Survey of MRI-Based Brain Tumor Segmentation Methods", ISSN 1007-0214 04/10 pp578595 Volume 19, Number 6, December 2014.

[6] R.Yogamangalam, B.Karthikeyan, "Segmentation Techniques Comparison in Image Processing", IJET, ISSN:0975-4024 Vol 5 No 1 Feb-Mar 2013.

[7] Eltaher Mohamed Hussein, Dalia Mahmoud Adam Mahmoud' "Brain Tumor Detection Using Artificial Neural Networks, Journal of Science and Technology Vol. 13, No. 2, December 2012.

[8] Kusum Rani, Reecha Sharma," Study of Different Image fusion Algorithm", International Journal of Emerging Technology and Advanced Engineering", (ISSN 22502459, Volume 3, Issue 5, May 2013). 
[9] Y. Zhang, L. Wu "An MR Brain Images Classifier via Principal Component Analysis and Kernel Support Vector Machine", Progress in Electromagnetics Research, Vol. 130, 369-388, august 2012.

[10] Bing Nan Li, Chee Kong Chui, Stephen Chang, S.H. Ong, "Integrating Spatial Fuzzy Clustering with level set methods for automates medical image segmentation", Computers in Medical and Imaging 41(2011) 1-10.

[11] Arashdeep Kaur, "An Automatic Brain Tumor Extraction System using Different Segmentation Methods", 2016 Second International Conference on Computational Intelligence \& Communication Technology.

[12] K. S. Angel Viji, Dr J. Jayakumari, "Modified Texture Based Region Growing Segmentation of MR Brain Images", 2013 IEEE Conference on Information and Communication Technologies (ICT 2013).

[13] Harneet Kaur, Sukhwinder Kaur,"Improved Brain Tumor Detection Using Object Based Segmentation”, (IJETT) - Volume 13 Number 1 - Jul 2014

[14] Ehab F. Badran, Esraa Galal Mahmoud, and Nadder Hamdy, "An Algorithm for Detecting Brain Tumors in MRI Images", 2010.

[15] B. Sathees, Dr. R. Anbu Selvi, "Feature Extraction Using Image Mining Techniques to Identify Brain Tumors", IEEE International Conference on ICIIECS' 15.

[16] Sudipta Roy, Sanjay Nag, Indra Kanta Maitra, Samir Kumar Bandyopadhyay, "A Review on Automated Brain Tumor Detection and Segmentation from MRI of Brain".

[17] Kusum Rani, Reecha Sharma, "Fusion of CT and MRI images using Discrete Multiwavelet Transform", International Journal of Computer Trends and
Technology (IJCTT) - ISSN: 2231-2803, volume4 Issue5-May 2013.

[18] Eman Abdel-Maksoud, Mohammed Elmogy, Rashid AlAwadi, "Brain tumor segmentation based on a hybrid clustering technique", Egyptian Informatics Journal (2015) 16,71-81, February 2015.

[19] Ilya Pollak, Alan S. Willsky, Hamid Krim, "Image Segmentation and Edge Enhancement with Stabilized Inverse Diffusion Equations". IEEE transactions on image processing, vol. 9, no. 2, February 2000.

[20] Lamia Jaafar Belaid, Walid Mourou, "Image segmentation: a Watershed transformation Algorithm", March 2009.

[21] M. Madheswaran and D. Anto Sahaya Dhas, "Classification of brain MRI images using support vector machine with various Kernels", ISSN 0970-938X- April 2015.

[22] Girja Sahu, P. Bhaiya, "A Survey Paper Based on the Classification of MRI Brain Images Using Soft Computing Techniques", IJETAE, ISSN 2250-2459, Volume 4, Issue 12, December 2014.

[23] Sun Hyung Kim, Vladimir Fonov, Joe Piven, John Gilmore, Clement Vachet, Guido Gerig, D. Louis Collins, Martin Styner,"Spatial Intensity Prior Correction for Tissue Segmentation in the Developing Human Brain", Proc IEEE Symp Biomed Imaging, 2011 :: 20492052 doi:10.1109 ISBI.2011.5872815.

[24] Mohammed Sabbih Hamoud Al-Tamimi, Ghazali Sulong, "Tumor Brain Detection Through Mr Images: A Review Of Literature", ISSN: 1992-8645, Vol. 62 No.2,2014. 